\title{
Neue Seminarreihe Führung und Management für Ärztinnen und Ärzte
}

Petra Ingenpass ${ }^{a}$

Beatrix Meyer $^{b}$

a Dr. med., stv. Leiterin Abteilung Tarife und Gesundheitsökonomie Spitalärzte

b Leiterin Abteilung Tarife und Gesundheitsökonomie Spitalärzte
Korrespondenz: FMH

Frohburgstrasse 15 CH-4600 Olten

Tel. 0313591111 Fax 0313591112 tarife.spital[at]fmh.ch
Aufgrund des zunehmenden Wettbewerbs im schweizerischen Spitalwesen steigen die Anforderungen an die Kaderärztinnen und -ärzte. Vertieftes ökonomisches Wissen ist immer mehr gefragt. Motivierte Mitarbeiter sind zudem für den Erfolg ebenso zentral wie eine gute Zusammenarbeit mit den Zuweisern. Darüber hinaus werden Gesprächssituationen anspruchsvoller - vor allem auch in rechtlich schwierigen Situationen.

Sehen Sie sich für diese Aufgaben ausreichend gewappnet? Verfügen Sie über die notwendigen Instrumente, um Ihren Fachbereich, Ihre Abteilung oder Ihre Klinik erfolgreich und souverän zu führen? Damit Sie zur Erfüllung dieser komplexen Ansprüche optimal gerüstet sind, bietet die FMH in Zusammenarbeit mit H+ Bildung eine Seminarreihe in vier Blöcken zu je zwei Tagen an. Hochkarätige Dozierende mit langjähriger Erfahrung vermitteln Ihnen in konzentrierter Form profundes Wissen. Die Teilnehmerzahl ist mehrheitlich auf 20 Personen beschränkt, damit interaktive Diskussionen möglich sind.

\section{Verfügen Sie über die notwendigen Instrumente, um Ihren Fachbereich, Ihre Abteilung oder Ihre Klinik erfolgreich und souverän zu führen?}

\section{Block I:}

\section{Medizin und Ökonomie}

(4./5.11.2014 in Aarau)

Im ersten Block werden Ihnen Instrumente und Konzepte der Betriebswirtschaftslehre vermittelt. Sie erfahren, wie Sie Ihren Fachbereich im Wettbewerb optimal ausrichten können und wie die Erlösverteilung innerhalb des Spitals erfolgt. Der Nutzen von Kennzahlen und DRG-Auswertungen wird ebenso aufgezeigt wie die Optimierung von Prozessen unter Wahrung guter Qualität.

\section{Block II:}

Projekt- und Veränderungsmanagement (20./21.1.2015 in Aarau)

Hier erfahren Sie, wie Sie die Unternehmensstrategie zielführend im eigenen Verantwortungsbereich umsetzen können. Darüber hinaus lernen Sie, welche Prinzipien und Vorgehensweisen im Veränderungsmanagement $\mathrm{zu}$ beachten sind. Zudem gewinnen einerseits der Umgang mit Stresssituationen sowie andererseits die Erhaltung der eigenen Leistungsbalance und diejenige der Mitarbeitenden immer mehr an Bedeutung.

\section{Block III:}

\section{eHealth und Zuweisermanagement}

(16./17.3.2015 in Aarau)

Wie kann die Spitalärzteschaft die vielfältigen Möglichkeiten von eHealth optimal für sich nutzen? Sie erfahren unter anderem, was beim elektronischen Datenaustausch zu beachten ist. Zudem lernen Sie, die Instrumente und die Methodik eines integrierten Zuweisermanagements für Ihren Verantwortungsbereich anzuwenden.

Block IV:

\section{Kommunikation und Führung}

(18./19.5.2015 in Aarau)

Welche Kommunikationsprinzipien führen für beteiligte Gesprächspartner zu guten Ergebnissen? Sie erlernen die Steuerung von verbalem und nonverbalem Kommunikationsverhalten - auch unter hohem zeitlichem und emotionalem Druck.

Sie erhalten für die gesamte Seminarreihe mit 25 Credits das Maximum der pro Jahr im Rahmen erweiterter Fortbildungen anrechenbaren Credits. Der Block I «Medizin und Ökonomie» kann auch getrennt gebucht werden, für diesen werden 14 Credits angerechnet.

Das ausführliche Programm finden Sie unter www.fmh.ch $\rightarrow$ Stationäre Tarife

Sichern Sie sich noch heute Ihren Platz wir freuen uns auf Sie! 